\title{
Genomic analysis of family data reveals additional genetic effects on intelligence and personality
}

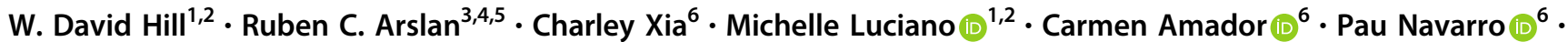 \\ Caroline Hayward $\mathbb{D}^{6} \cdot$ Reka Nagy $^{6} \cdot$ David J. Porteous $\mathbb{D}^{1,7,8} \cdot$ Andrew M. McIntosh $\mathbb{D}^{1,9} \cdot \operatorname{lan}$ J. Deary ${ }^{1,2}$. \\ Chris S. Haley ${ }^{6,10} \cdot$ Lars Penke ${ }^{1,3,4}$
}

Received: 6 February 2017 / Revised: 8 November 2017 / Accepted: 21 November 2017 / Published online: 10 January 2018

(c) The Author(s) 2018. This article is published with open access

\begin{abstract}
Pedigree-based analyses of intelligence have reported that genetic differences account for $50-80 \%$ of the phenotypic variation. For personality traits these effects are smaller, with $34-48 \%$ of the variance being explained by genetic differences. However, molecular genetic studies using unrelated individuals typically report a heritability estimate of around $30 \%$ for intelligence and between 0 and $15 \%$ for personality variables. Pedigree-based estimates and molecular genetic estimates may differ because current genotyping platforms are poor at tagging causal variants, variants with low minor allele frequency, copy number variants, and structural variants. Using 20,000 individuals in the Generation Scotland family cohort genotyped for 700,000 single-nucleotide polymorphisms (SNPs), we exploit the high levels of linkage disequilibrium (LD) found in members of the same family to quantify the total effect of genetic variants that are not tagged in GWAS of unrelated individuals. In our models, genetic variants in low LD with genotyped SNPs explain over half of the genetic variance in intelligence, education, and neuroticism. By capturing these additional genetic effects our models closely approximate the heritability estimates from twin studies for intelligence and education, but not for neuroticism and extraversion. We then replicated our finding using imputed molecular genetic data from unrelated individuals to show that $\sim 50 \%$ of differences in intelligence, and $\sim 40 \%$ of the differences in education, can be explained by genetic effects when a larger number of rare SNPs are included. From an evolutionary genetic perspective, a substantial contribution of rare genetic variants to individual differences in intelligence, and education is consistent with mutation-selection balance.
\end{abstract}

W. David Hill, Ruben C. Arslan and Charley Xia contributed equally to this work.

Electronic supplementary material The online version of this article (https://doi.org/10.1038/s41380-017-0005-1) contains supplementary material, which is available to authorised users.

W. David Hill

David.Hill@ed.ac.uk

1 Centre for Cognitive Ageing and Cognitive Epidemiology, University of Edinburgh, 7 George Square, Edinburgh EH8 9JZ, UK

2 Department of Psychology, University of Edinburgh, 7 George Square, Edinburgh EH8 9JZ, UK

3 Georg Elias Müller Institute of Psychology, Georg August University Göttingen, Göttingen, Germany

4 Leibniz Science Campus Primate Cognition, Göttingen, Germany

5 Center for Adaptive Rationality Max Planck Institute for Human Development Lentzeallee 94, 14195 Berlin, Germany

\section{Introduction}

The scores from different types of cognitive ability tests correlate positively and the variance that is shared between tests is termed general intelligence, general cognitive ability, or $g$ [1]. General intelligence typically accounts for

6 MRC Human Genetics Unit, Institute of Genetics and Molecular Medicine, University of Edinburgh, Edinburgh, UK

7 Generation Scotland, Centre for Genomic and Experimental Medicine, Institute of Genetics and Molecular Medicine, University of Edinburgh, Edinburgh EH4 2XU, UK

8 Medical Genetics Section, Centre for Genomic and Experimental Medicine, Institute of Genetics and Molecular Medicine, University of Edinburgh, Edinburgh, UK

9 Division of Psychiatry, University of Edinburgh, Royal Edinburgh Hospital, Edinburgh EH10 5HF, UK

10 The Roslin Institute and Royal (Dick) School of Veterinary Sciences, University of Edinburgh, Edinburgh, UK 
around $40 \%$ of the overall variance among humans in batteries that contain tests of diverse cognitive abilities. The personality traits of extraversion and neuroticism are two of the five higher-order personality factors that are consistently identified in dimensional models of personality. High levels of extraversion are associated with positive affectivity and a tendency to engage with, and to enjoy, social situations. High levels of neuroticism are associated with stress sensitivity, as well as mental and physical disorders [2]. All of these traits are partly heritable, but have also been linked to evolutionary fitness. This paradox, that cognitive ability and personality appear to be under selective pressure yet retain heritable variation, could be resolved if rare variants, which are less amenable to selection, are found to play a major role in the genetic contribution to variance in these traits. We test whether genetic variants not in LD with genotyped single-nucleotide polymorphisms (SNPs) (including rare variants, copy number variants (CNVs) and structural variants) make a contribution to intelligence and personality differences using two separate methods.

Firstly, using a recently developed analytic design for combined pedigree and genome-wide molecular genetic data, we test whether rare genetic variants, $\mathrm{CNVs}$, and structural variants make an additional contribution to the genetic variance in intelligence, neuroticism, and extraversion. Secondly, using unrelated individuals, and genotype data imputed using the Haplotype Reference Consortium [3, 4] (HRC) data, we use minor allele frequency (MAF) stratified GREML (GREML-MS) to quantify the effect of SNPs with a MAF of $\geq 0.001$ to determine if this additional variance can also be recovered based on SNPs alone using imputation.

General intelligence has been found to be heritable, with twin and family studies estimating that 50 to $80 \%$ [5] of phenotypic variance is due to additive genetic factors, a proportion that increases with age from childhood to adulthood [6]. Heritability can also be estimated from molecular genetic data. Using the genomic-relatedness-matrix restricted maximum likelihood single component (GREML-SC) method, the additive effects of common SNPs are estimated to collectively explain between 20 and $50 \%$ of variation in general intelligence [7, 8], with an estimate of around $30 \%$ in the largest studies [9]. General intelligence is also a significant predictor of fitness components including mortality [10], fertility [11, 12] and higher social status [13], as well as mental and physical disease [6]. General intelligence is associated with developmental stability $[14,15]$, suggesting that it is not selectively neutral.

As directional selective pressure on a trait is expected to deplete its genetic variation, the existence of such robust heritability findings seems paradoxical when evolutionary theory is considered [16]. However, mutation-selection balance provides an explanation of how genetic variation can be maintained for quantitative traits that are under directional selective pressure. Mutation-selection balance describes instances where mutations that are deleterious to the phenotype occur within a population at the same rate that they are removed through the effects of selective pressure. Due to the removal of variants with deleterious effects on the phenotype, the existence of common variants with medium to large effects is not expected under mutation-selection balance. This is consistent with the current findings from large genome-wide association studies (GWAS) on cognitive phenotypes, including general intelligence and education, where common SNPs collectively explain a substantial proportion of phenotypic variance, but the individual effect size of each genome-wide significant SNP discovered so far is around $0.02 \%[17,18]$.

Population genetic simulations show that very rare (MAF $<0.1 \%)$ variants explain little of the population variance in traits that are not under selection [19]. However, the contribution made by rare variants increases when their effects on a trait and on fitness are correlated either through pleiotropy, or by the trait directly affecting fitness [19]. The genetically informative evidence that is available tends to show that variants associated with intelligence are also linked to better health [20,21], although these effects may be outweighed by a negative effect on fertility [22, 23]. There is also evidence that the regions of the genome making the greatest contribution to intelligence differences have undergone purifying selection [24]. Whereas this does not necessarily imply that intelligence has been selected for or against across our evolutionary history, it does indicate that genetic variants that are associated with intelligence are also associated with fitness, which suggests that rare genetic variants and hence mutation-selection balance, may act to maintain intelligence differences [19].

Empirical studies so far have failed to find evidence of a link between intelligence and rare variants [25]. These studies have often been limited in scope, with only CNVs or exonic regions being considered, or being limited in statistical power because all rare variants were treated as having the same direction of effect through the use of burden tests [25-29]. Where such tests have found an association these have been in small samples and subsequently failed to replicate [30]. However, in large samples, rare variants found within regions of the genome under purifying selection have been found to be associated with educational success [31], an effect that was greater for genes expressed in the brain. Hence, rare variants found in some genes appear to have an effect on intelligence.

Less is known about the genetics of personality [32]. As with intelligence, heritability estimates for extraversion and neuroticism are much higher, around 34-48\%, when based on quantitative (twin- and family-based) genetic methods [33] compared to molecular genetic estimates $(4-15 \%$ for neuroticism [34] and 0-18\% for extraversion [35, 36]). 
Both extraversion and neuroticism are predictive of social and behavioural outcomes as well as anxiety, well-being, and fertility [37-40]. Positive genetic correlations have been reported for extraversion with attention deficit hyperactivity disorder and bipolar disorder, and for neuroticism with depression and anorexia nervosa [36].

In the current study, we quantify the total genetic effect across the autosomes on intelligence (including education, which shows strong genetic correlations with general intelligence [41] and is used as a proxy-phenotype for it in genetic studies [42]), extraversion and neuroticism. Two recent approaches allow us to include genetic variation not normally captured using GWAS. Firstly, as our sample included nominally unrelated individuals with varying degrees of genetic similarity, as well as family members who all provided genome-wide SNP data, we were able to decompose two genetic sources of variance corresponding to genetic effects associated with common SNPs at the population level $\left(h_{\mathrm{g}}^{2}\right)$, and genetic effects associated with kinship $\left(h_{\text {kin }}^{2}\right)$ (i.e., associated with SNPs on a family basis). Among related individuals, LD is stronger and hence allows us to capture variation not tagged by common SNPs at the population level. This includes rare variants, CNVs, and other structural variants. As the inclusion of family members can introduce confounding between shared genetic effects and shared environmental effects [43], we use the GREML-KIN method by Xia and colleagues [44] to control for sibling effects, spouse effects and family effects. By using information from both nuclear family relationships and the many more distant pedigree relationships in the cohort we analyse, this novel approach allows us to estimate kin-specific genetic variation net of common environmental effects. Secondly, we validate the findings for intelligence and education using unrelated individuals by using genotypes imputed using the HRC panel [4]. By using GREML-MS to derive a heritability estimate we were able include rare SNPs (MAF 0.001-0.01) as well as partition the SNPs by MAF to determine the contribution made to trait variation by rare variants.

\section{Materials and methods}

\section{Samples}

Data was used from the Generation Scotland: Scottish Family Health Study (GS:SFHS) [4, 45, 46]. A total of $24,090 \quad$ individuals $\quad\left(N_{\text {male }}=9927, \quad N_{\text {female }}=14,163\right.$, Age $_{\text {mean }}=47.6$ ) were sampled from Glasgow, Tayside, Ayrshire, Arran and North-East Scotland of whom 23,919 donated blood or saliva for DNA extraction. These samples were collected, processed, and stored using standard procedures and managed through a laboratory information management system at the Wellcome Trust Clinical
Table 1 Degree of relatedness in the 20,032 GS:SFHS data and number of pair-wise relationships

\begin{tabular}{ll}
\hline Matrix & $\begin{array}{l}\text { Number of non-zero } \\
\text { off-diagonal entries }\end{array}$ \\
\hline GRM $_{\mathrm{g}}$ & $200,630,496$ \\
$\mathrm{GRM}_{\text {kin }}$ & 41,174 \\
$\mathrm{SRM}_{\text {Family }}$ & 20,115 \\
$\mathrm{SRM}_{\text {Sibling }}$ & 1767 \\
SRM $_{\text {Couple }}$ & 8495 \\
Degree of relationship & Number of pairs \\
1st degree & 18,320 \\
2nd degree & 7851 \\
3rd degree & 4129 \\
4th degree & 3950 \\
5th degree & 11,032 \\
Unrelated individuals & $200,585,162$ \\
\hline
\end{tabular}

For the $\mathrm{G}$ matrix all off-diagonal entries are non-zero

The distance of the relationship is identified using SNP relatedness and according to approximate ranges of the expected pair-wise relatedness, $0.5^{i-0.5}$ to $0.5^{i+0.5}$ for $i^{\text {th }}$ degree relatives

Unrelated individuals defined as more than 5th degree relatives $r \leq 0.022$

Research Facility Genetics Core, Edinburgh [47]. The yield of DNA was measured with a PicoGreen and normalised to $50 \mathrm{ng} / \mu \mathrm{l}$ prior to genotyping. Genotype data were generated using an Illumina Human OmniExpressExome -8- v1.0 DNA Analysis BeadChip and Infinium chemistry [48]. We then used an identical quality control procedure as Xia et al. [44]. that included removing SNPs not on autosomes or with a MAF of $<0.05$, a Hardy-Weinberg Equilibrium $P$ value $<10^{-6}$, and a missingness of $>5 \%$. This left 519,729 common SNPs from 22 autosomes. Following quality control, a total of 20,032 genotyped individuals $\left(N_{\text {female }}=\right.$ $11,804)$ were retained; 18,293 of these individuals were a part of 6578 nuclear or extended families [49]. The mean age of the sample was 47.4 years $(\mathrm{SD}=15.0$, range 18 to 99 years). The degree of the relationships found in GS: SFHS as well as the size of each of the matrices can be found in Table 1.

\section{Ethics}

The Tayside Research Ethics Committee (reference 05/ S1401/89) provided ethical approval for this study.

\section{Phenotypes}

General intelligence $(g)$, years in education (Education), neuroticism, and extraversion were examined using GREML-KIN, and GREML-MS. Four cognitive tests were 
used to derive general intelligence; the Mill Hill Vocabulary Scale (MHVS) (test re-test reliability over 2 years 0.90 , split half reliability $r=0.90)[50,51]$, the Wechsler Digit Symbol Substitution Task (DST) (test re-test reliability $r=.90$ ) [52], Wechsler Logical Memory, which measures Verbal declarative memory (split half reliability, part $1=0.88$, part two 0.79) [53] and executive function (phonemic Verbal fluency, using letters C, F, L) (Cronbach's alpha $=0.83)$ [54]. The general factor of intelligence $(g)$ was derived by extracting the first unrotated principal component from the four cognitive tests. This single component accounted for $42.3 \%$ of the variance in the total sample and each of the individual tests used demonstrated strong loadings on the first unrotated component (DST 0.58, Verbal Fluency 0.72, MHVS 0.67 and Verbal declarative memory 0.63). Education was calculated in the GS:SFHS as the years of full time formal education, which was recoded into an ordinal scale from 0 to 10 (0: 0 years, $1: 1-4$ years, 2: 5-9 years, 3: 10-11 years, 4: 12-13 years, 5: 14-15 years, 6: 16-17 years, 7: 18-19 years, 8: 20-21 years, 9: 22-23 years, 10: $>24$ years of education). Education and general intelligence were positively correlated $(r=0.38$, $\left.\mathrm{SE}=0.01, P<2.20 \times 10^{-16}\right)$.

The other two measures examined were the personality traits of extraversion and neuroticism, which were measured using the Eysenck Personality Questionnaire Revised Short Form, a self-report questionnaire requiring a yes or no response to 24 items [55]. Both scales have reliabilities of Cronbach's alpha $>0.85$ [55].

The effects of age, sex and population stratification were adjusted for using regression prior to fitting the models in GREML. Supplementary Fig. 1 shows the number of principal components used to control for population stratification for each of the phenotypes used.

\section{Statistical method}

\section{GREML-KIN: partitioning phenotypic variance into five components}

For each of the phenotypes examined here, variance was partitioned into five corresponding effects plus residual variance. This variance components analysis is based on the work of Zaitlen and colleagues [43] who developed a method for estimating two genetic sources of variance in a data set with a measured family structure. Firstly, the variance component $\mathrm{G}$ can be estimated and used to derive $h_{\mathrm{g}}^{2}$, the proportion of phenotypic variance explained by common SNPs, and secondly, the additional genetic effects associated with pedigree can be captured by $\mathrm{K}$ and used to derive $h_{\text {kin }}^{2}$, the proportion of phenotypic variance that is explained by genetic effects that are clustered within families. More recently this method has been extended by
Xia and colleagues [44] to adjust for similarity between siblings, spouses and nuclear family members. We refer to the extended method as GREML-KIN. The two genetic matrices described by Zaitlen et al. and Xia et al. model the effects associated with common SNPs $\left(h_{\mathrm{g}}^{2}\right)$ at the population level and those associated with pedigree $\left(h^{2}\right.$ kin $)$, respectively. These two genetic sources of variance were quantified using a genetic relationship matrix (GRM) derived in the GCTA software [56].

\section{Matrix construction}

Genetic matrices A genomic relationship matrix $\left(\mathrm{GRM}_{\mathrm{g}}\right)$ was used to derive the variance component of $\mathrm{G}$ in order to quantify the contribution made by common SNPs, $h_{\mathrm{g}}^{2}$. This was derived in the manner set out by Yang and colleagues [56], where the estimated genomic relatedness between each pair of individuals is derived from identity by state SNP relationships and is found in each off-diagonal entry in the GRM). As the variance attributable to the shared environment was explicitly modelled here, no relationship cutoff (typically, 0.025 is used) was applied to the GRM.

$$
\frac{1}{N} \sum_{i=1}^{N} \frac{\left(x_{j i}-2 p_{i}\right)\left(x_{k i}-2 p_{i}\right)}{2 p_{i}\left(1-p_{i}\right)}
$$

MAF for SNP $i$ is denoted as $p i$ and the allelic dose $(x)$ for individuals $j$ or $k$ at locus $i$ is described as $x_{j i}$ or $x_{k i}$. N indicates the total number of SNPs.

The kinship relationship matrix, $\mathrm{GRM}_{\mathrm{kin}}$, (used to derive the variance component $K$ ) was derived using the method described by Zaitlen et al. [43] by modifying the $\mathrm{GRM}_{\mathrm{g}}$. Here, values in the $\mathrm{GRM}_{\mathrm{g}}$ that were equal to or $<0.025$ were set to 0 .

Similarity matrices Three similarity matrices (SRM) were used to capture the variance associated with specific relationships between individuals. Each SRM was created by deriving an $N$ by $N$ matrix (where $N$ is number of individuals) with diagonal entries set to 1 and non-diagonal entries set to 1 if the pair of individuals have the relationship described, or set to zero otherwise. The three SRMs derived here captured variance associated with the similarity of spouses, $\left(\mathrm{SRM}_{\text {Couple }}\right.$, similarity variance component $\mathrm{C}$ ), siblings $\left(\mathrm{SRM}_{\mathrm{Sibling}}\right.$, similarity variance component $\left.\mathrm{S}\right)$ and nuclear families $\left(\mathrm{SRM}_{\text {Family }}\right.$, similarity variance component F). As discussed by Xia et al. [44], whereas these matrices are not formed using genetic data, the $\mathrm{SRM}_{\text {Couple }}$ will very likely will capture some effects of assortative mating, which can be couples who, before meeting, were more similar than chance in terms of the trait under investigation, as well as couples who become more similar due to time spent 
Table 2 Results of variance components analyses for cognitive abilities and personality from the full model and the final model selected in a stepwise selection procedure

\begin{tabular}{|c|c|c|c|c|c|c|c|c|}
\hline Phenotype & $N$ & Model & $\begin{array}{l}\text { Variance } \\
\text { components }\end{array}$ & $\begin{array}{l}\mathrm{GRM}_{\mathrm{g}} \\
h_{\mathrm{g}}^{2} \% \text { (S.E.) }\end{array}$ & $\begin{array}{l}\mathrm{GRM}_{\text {kin }} \\
h_{\text {kin }}^{2} \% \text { (S.E.) }\end{array}$ & $\begin{array}{l}\text { SRM }_{\text {Family }} \\
e_{\mathrm{f}}^{2} \% \text { (S.E.) }\end{array}$ & $\begin{array}{l}\text { SRM }_{\text {Sibling }} \\
e_{\mathrm{s}}^{2} \% \text { (S.E.) }\end{array}$ & $\begin{array}{l}\text { SRM }_{\text {Couple }} \\
e_{\mathrm{c}}^{2} \% \text { (S.E.) }\end{array}$ \\
\hline \multicolumn{9}{|l|}{ Cognitive } \\
\hline \multirow[t]{2}{*}{$g$} & 19,036 & Full & GKFSC & $21.1(2.0)$ & $41.5(4.8)$ & $1.0 \times 10^{-4}(2.2)$ & $8.9(1.3)$ & $26.4(2.6)$ \\
\hline & 19,036 & Selected & GKSC & $22.7(2.1)$ & $31.3(2.9)$ & - & $9.2(1.3)$ & $22.1(2.0)$ \\
\hline \multirow[t]{2}{*}{ Education } & 18,528 & Full & GKFSC & $13.3(2.0)$ & $39.4(5.1)$ & $1.0 \times 10^{-4}(2.4)$ & $10.9(1.4)$ & $36.1(2.7)$ \\
\hline & 18,528 & Selected & GKSC & $15.6(2.1)$ & $28.1(3.0)$ & - & $11.4(1.4)$ & $31.3(2.8)$ \\
\hline \multicolumn{9}{|l|}{ Personality } \\
\hline \multirow[t]{2}{*}{ Neuroticism } & 19,494 & Full & GKFSC & $10.7(2.0)$ & $14.9(5.1)$ & $2.3(2.5)$ & $1.0 \times 10^{-4}(1.4)$ & $1.0 \times 10^{-4}(3.4)$ \\
\hline & & Selected & GK & $10.8(2.0)$ & $19.2(2.5)$ & - & - & - \\
\hline \multirow[t]{2}{*}{ Extraversion } & 19,487 & Full & GKFSC & $11.3(2.0)$ & $4.9(5.1)$ & $7.3(2.5)$ & $1.0 \times 10^{-4}(1.4)$ & $1.0 \times 10^{-4}(3.3)$ \\
\hline & 19,487 & Selected & GF & $13.0(1.7)$ & - & $9.0(1.1)$ & - & - \\
\hline
\end{tabular}

together. Dominance effects can be captured with the

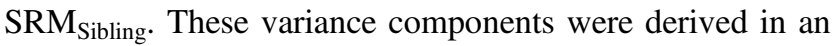
identical manner to Xia et al. [44], however, we have changed the matrices' names to reflect that they may also capture non-additive genetic influences, as well as the effects of assortative mating.

Estimating the phenotypic variance explained For each trait we first fitted the two GRMs and the three SRMs simultaneously using a linear mixed model (LMM) using the GCTA software [56, 57]. This full model is referred to as the GKFSC model, as it includes the genetic, kinship, family, sibling and couple matrices.

$$
Y=G+K+F+S+C+\varepsilon
$$

Here, $\boldsymbol{Y}$ is a vector of standardised residuals derived from one of the phenotypes. Random genetic effects were explained by fitting the $\mathrm{G}$ and $\mathrm{K}$, which captured variants in LD with common SNPs found across a population and the extra genetic effects captured by the increase in LD found between members of the same extended family, respectively. Similarity between related pairs of individuals was adjusted for by fitting the F, S and $\mathrm{C}$ to control for the contributions made by environmental similarities, as well as any effect of assortative mating and dominance, that could result in members of a nuclear family, siblings, and couples, being more similar. This enabled us to extract the variance associated with these matrices from our genetic matrices to ensure that the genetic estimates derived are not biased by these confounding influences.

Restricted maximum likelihood (REML), implemented using the GCTA software [56], was used to estimate the variance explained by each of the variance components, with statistical significance determined using a loglikelihood ratio test (LRT) and the Wald test. Model selection began with the full GKFSC model (referred to as the full model). Components were dropped if they were not statistically significant according to both the Wald and the LRT tests. The model that contained only components that explained a significant proportion of variance is referred to as the selected model. If more than one component could be dropped from the model, we dropped the one with the worse fit first and then tested the significance of the other. The full results of each model can be seen in Supplementary Table 1. The phenotypic variance explained by the variance components of $\mathrm{G}, \mathrm{K}, \mathrm{S}, \mathrm{F}$ and $\mathrm{C}$ used to derive $h_{\mathrm{g}}^{2}$ (common SNP-associated effects), $h_{\text {kin }}^{2}$ (pedigree-associated genetic effects), $e_{\mathrm{f}}^{2}$ (family effects), $e_{\mathrm{s}}^{2}$ (sibling effects) and $e_{\mathrm{c}}^{2}$ (couple effects) were estimated (Table 2).

Interpretation of the variance explained by GREMLKIN Despite collinearity between the five matrices, simulations conducted by Xia et al. [44] show that this method provides robust results due to the dense relationships within the GS:SFHS cohort. The GS:SFHS is a family-based cohort and the participants are related to varying degrees, including 1767, 18,320, 7851, 4129, 3950 and 11,032 pairs of couples, 1st, 2nd, 3rd, 4th, and 5th degrees of relatives, respectively. Therefore, what is shared between the $\mathrm{SRM}_{\text {Family }}$ matrix and $\mathrm{GRM}_{\text {kin }}$ matrix is information on the $\sim 18 \mathrm{k} 1$ st degree relatives. However, $\mathrm{SRM}_{\text {Family }}$ holds $\sim 1.8 \mathrm{k}$ pairs of unique entries (couple pairs) and $\mathrm{GRM}_{\text {kin }}$ holds $\sim 23 \mathrm{k}$ pairs of unique entries (equivalent $2 \mathrm{nd}-5$ th degree relative pairs of who were greater than 0.025 genetically identical). The unique entries from both matrices result in an increase of power, which allows the disentangling of the variance from those two different sources.

An additional point is that the pedigree-associated genetic effects decay as the distance of the relationship increases, whereas nuclear family similarity effects do not. Thus, the fact that GS:SFHS consist of different classes of relatives, 
as well as the unique entries within the $\mathrm{GRM}_{\text {kin }}$ and

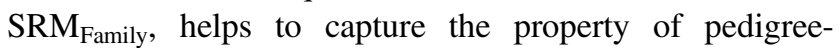
associated genetic variants. This logic extends to separating the variance from each of the similarity matrices. Although $\mathrm{SRM}_{\text {Couple }}$ and $\mathrm{SRM}_{\mathrm{Sib}}$ are nested within the $\mathrm{SRM}_{\text {Family, }}$ there are 9853 pairs of unique entries (representing parents-

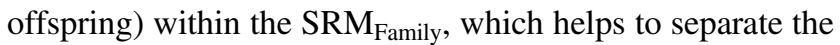
similarity matrices. As shown by Xia et al. [44], this method reliably identifies the major sources of variance that contribute to trait architecture in a realistic simulation of confounding. However, as with any method, effects become harder to detect as significant as they become smaller, since more power is needed for the reliable detection of small signals. This means that if one of the matrices only contributes to a small proportion of the overall phenotypic variance (e.g., $<5 \%$ in GS:SFHS) the component will be dropped in the model selection procedure as it will not attain statistical significance. The small effects from the excluded component will have only a limited influence on the estimates of the major components that are retained in the final model. Thus, the major components we detected for each trait should be estimated reliably.

An additional caveat of the GREML-KIN method is that it requires a cohort of related individuals where there are a sufficient number of different degrees of relatives and family members so that pedigree genetic effects can be disentangled from the family environmental influences. Due to these requirements, a simulation study should be conducted in a data set before using GREML-KIN. The goal of these simulations is to examine whether there are a suitable level of appropriate relationships, and whether the confounding environmental and genetic factors can be discomposed accurately in the population under investigation as was shown by Xia et al. in the $\sim 20,000$ members of GS:SFHS.

In the current study, we computed $\mathrm{SRM}_{\mathrm{Sib}}$, $\mathrm{SRM}_{\text {Family, }}$, and $\mathrm{SRM}_{\text {Couple }}$ to measure the similarity shared between siblings, nuclear family members, and couples, respectively. The similarity shared between siblings is a product of additive genetic effects and dominance genetic effects, in addition to any environmental influences. However, as $\mathrm{GRM}_{\mathrm{g}}$ and $\mathrm{GRM}_{\mathrm{kin}}$ capture additive genetic effects, only environmental influences and some of the total dominance genetic effect remain in $\mathbf{S R M}_{\mathrm{Sib}}$. Similarly, $\mathbf{S R M}_{\mathrm{Family}}$ models similarity between nuclear family members, which is composed of additive genetic effects and environmental factors. In the presence of $\mathrm{GRM}_{\mathrm{g}}$ and $\mathrm{GRM}_{\mathrm{kin}}$, what remained in $\mathrm{SRM}_{\text {Family }}$ will be variance attributable to environmental factors. The $\mathrm{SRM}_{\text {Couple }}$ represents the similarity between couples, which can be due to assortative mating and shared environmental influences.
GREML-MS analysis In order to show that the $\mathrm{GRM}_{\text {kin }}$ in GREML-KIN captures the contributions made by genetic variants poorly tagged by genotyped SNPs and is not confounded by the inclusion of close relatives, we replicated our results using unrelated individuals. Using genotyped data, imputed using the Haplotype Consortium (HRC) [3, 4] data set, allowed investigation into low-frequency variants using the Sanger Imputation Service (https://imputation.sa nger.ac.uk/). A quality control check was performed by checking autosomal haplotypes to ensure that strand orientation, reference allele and position matched the reference panel. Data were then pre-phased using the Shapeit2 duohmm option provided by the Shapeit2 v2r837 software [58-60], where the family structure of GS: SFHS was used to improve the imputation quality [61]. Finally, an imputation quality score of info $<0.4$ was used to exclude poorly imputed variants and non-bi-allelic variants. This resulted in 11,497 491 bi-allelic SNPs with MAF $>0.001$ available for analysis.

A relatedness cutoff was applied to the participants of GS: SFHS of 0.025 resulting in a sample size of 7370 . Note, that relatedness was based on the $\mathrm{GRM}_{\mathrm{g}}$, -i.e., estimated by using all genotyped common SNPs on the autosomes for the whole population. To test whether the additional variance captured by our $\mathrm{GRM}_{\mathrm{kin}}$ is due to less common variants, imputed and genotyped variants were assigned to one of six matrices describing the frequency of the minor allele. The six bins, and the matrices derived using them, were $\mathrm{MAF}=0.001-0.01$ $\left(\mathrm{GRM}_{0.001-0.01}\right), \mathrm{MAF}=>0.01-0.1\left(\mathrm{GRM}_{0.01-0.1}\right), \mathrm{MAF}=>$ $0.1-0.2 \quad\left(\mathrm{GRM}_{0.1-0.2}\right), \quad \mathrm{MAF}=>0.2-0.3 \quad\left(\mathrm{GRM}_{0.2-0.3}\right),>$ $0.3-0.4\left(\mathrm{GRM}_{0.3-0.4}\right), \mathrm{MAF}=>0.4-0.5\left(\mathrm{GRM}_{0.4-0.5}\right)$ [62]. These six matrices were then fitted simultaneously and analysed using REML.

\section{Results}

The results of the full GKFSC models as well as the results of the selected models, can be seen in Table 2. For general intelligence $(g)$ the final model was the GKSC model, allowing for a significant contribution from additive common genetic effects, additive pedigree-associated genetic variants, sibling similarity and couple similarity. For $g$, common SNPs $\left(h_{\mathrm{g}}^{2}\right)$ explained $23 \%(\mathrm{SE}=2 \%)$ of the phenotypic variation. Pedigree-associated genetic variants $\left(h^{2}\right.$ kin $)$ added an additional $31 \%(\mathrm{SE}=3 \%)$ to the genetic contributions to $g$, yielding a total contribution of genetic effects of $54 \%$ ( $\mathrm{SE}=3 \%)$ on $g$. The sibling effects $\left(e_{\mathrm{s}}^{2}\right)$ and couple effects $\left(e_{\mathrm{c}}^{2}\right)$, accounted for $9 \%(\mathrm{SE}=1 \%)$ and $22 \%$ $(\mathrm{SE}=2 \%)$, respectively. As noted previously, these estimates could also include effects of dominance and assortative mating, respectively. 


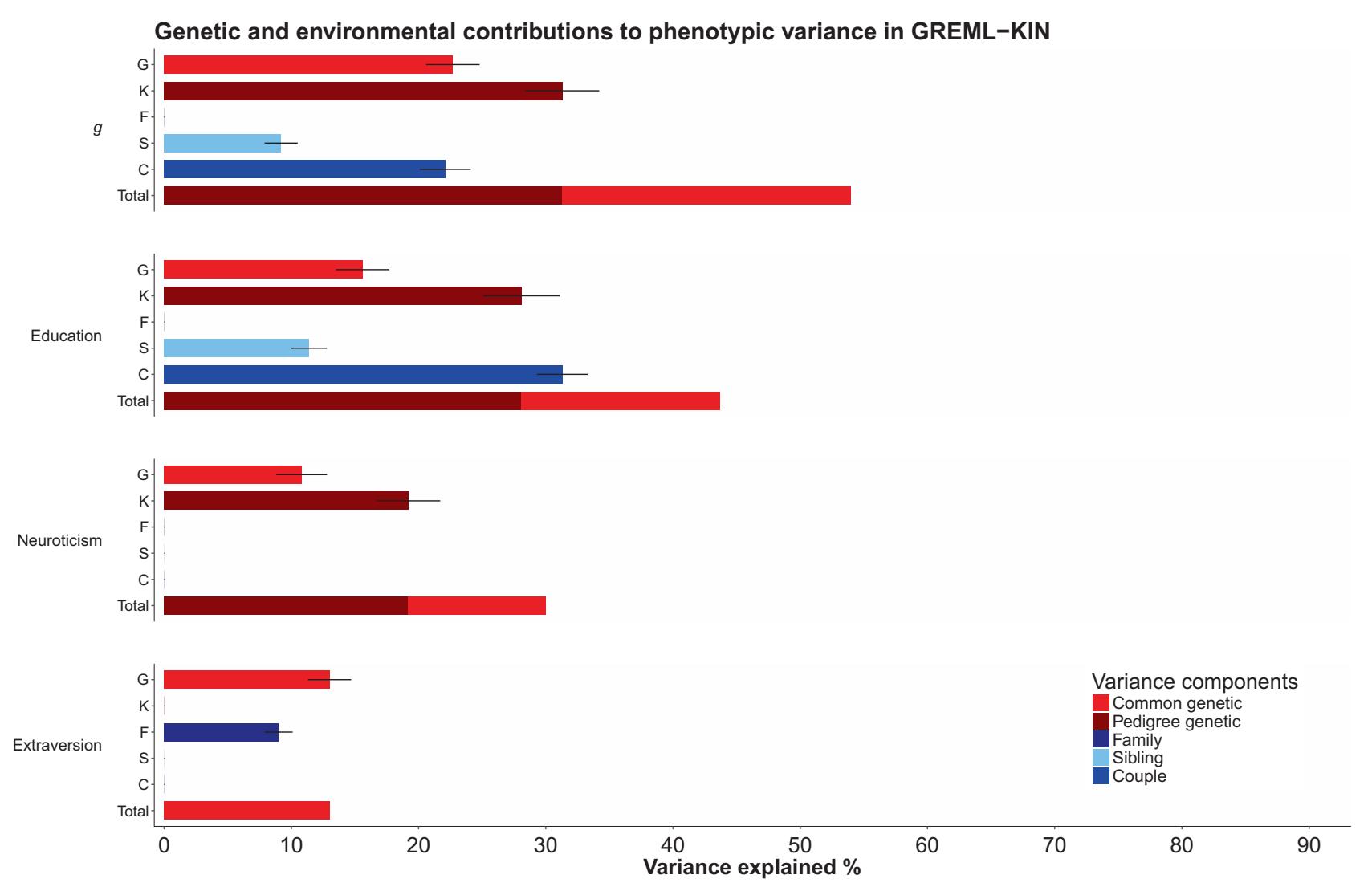

Fig. 1 Genetic contribution to each phenotype using the selected models plotted for each of the phenotypes. Each component from the selected models is plotted individually, with the stacked bar plot showing the total proportion of the variance explained by genetic factors in the selected models. Error bars indicate standard errors

GKSC model. The C component did not attain statistical significance for logical memory with the selected model being GKS.

For neuroticism the final model consisted of contributions from the variance components $\mathrm{G}$ and $\mathrm{K}$. Additive common genetic effects explained $11 \%(\mathrm{SE}=2 \%)$ of the variance with pedigree-associated variants explaining an additional $19 \%(\mathrm{SE}=3 \%)$. The $\mathrm{F}, \mathrm{S}$, and $\mathrm{C}$ components were not statistically significant and the family similarity component accounted for only $2 \%$ of the variance in the full model and $1 \%$ in a model that included only the $\mathrm{G}$ and the $\mathrm{K}$ in addition to $\mathrm{F}$.

For extraversion the only detectable source of genetic variation came from the $\mathrm{G}$, which accounted for $13 \%$ ( $\mathrm{SE}=$ $2 \%)$, with $\mathrm{F}$ explaining a further $9 \% \quad(\mathrm{SE}=1 \%)$ of the phenotypic variation. The lack of pedigree-associated genetic effects could be due to low statistical power, as $\mathrm{K}$ explained $5 \%$ of the variance in the full model and $6 \%$ in a GKF model, but with a relatively large SE, estimated at $5 \%$.

In addition to our model selection procedure, we also fit all possible component combinations for all phenotypes, to

The selected model for the Mill Hill Vocabulary test, the Verbal Fluency test and Digit Symbol test was the 
Comparison between molecular genetic and family-based pedigree estimates

$g$

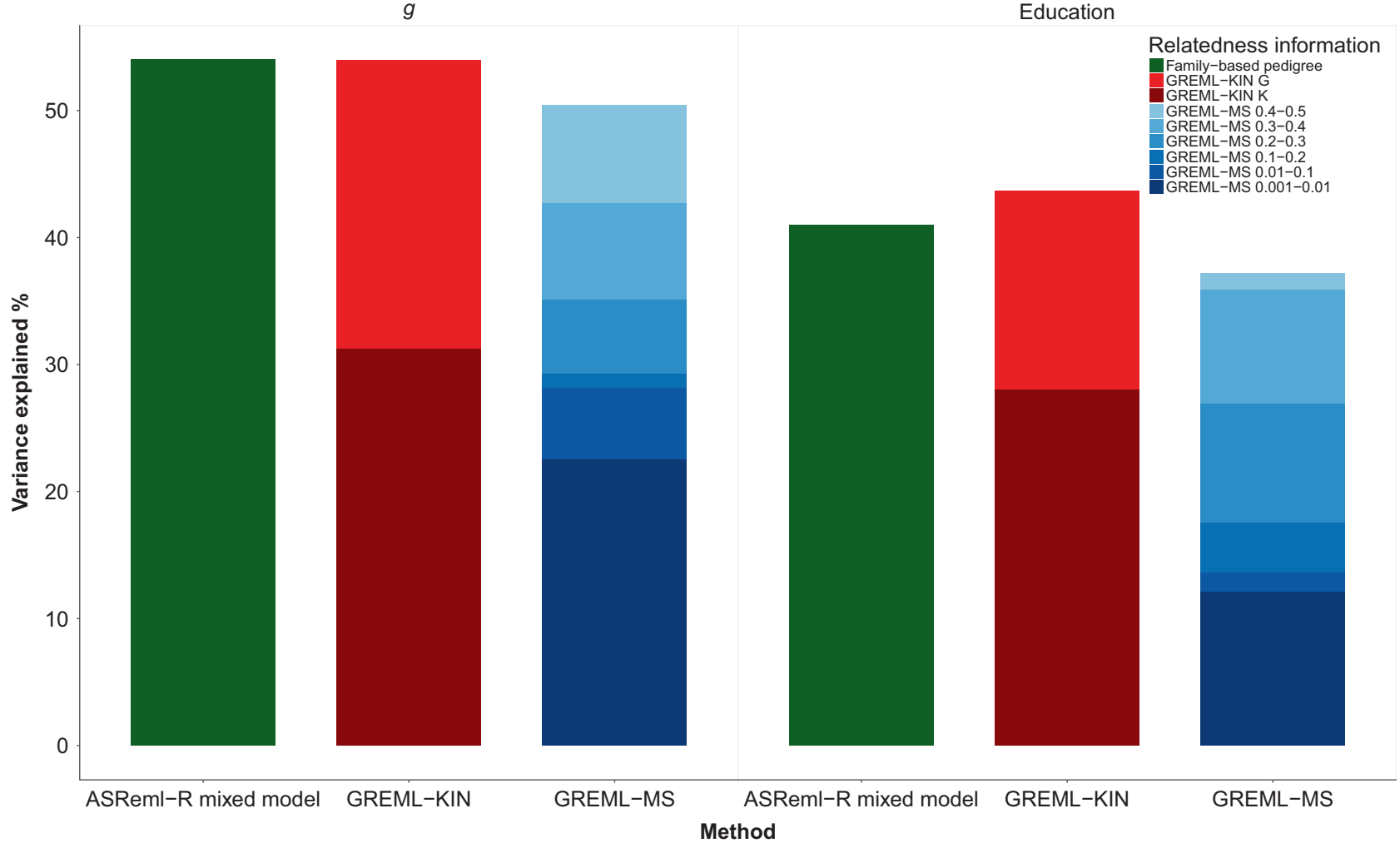

Fig. 2 Bar plots showing the proportion of variance explained using family-based methods and using molecular genetic data in related and unrelated samples. All of these analyses were performed using the same GS:SFHS data $(n=20,522$, Education $n=22,406)$. Using related individuals and GREML-KIN, a sample size of 19,036 was available for general intelligence, and 18,528 for education after quality control. GREML-MS was conducted on unrelated individuals using a sample of $n=7019$ for general intelligence and 6860 for Education. Estimates depicted in red were derived in the current study using GREML-KIN and show two sources of genetic

show a more complete account of the data and to give readers the ability to explore the consequences of including different components for the results, even when some of those components were not significant. The results have been made interactively available at https://rubenarslan. github.io/generation_scotland_pedigree_gcta/.

The results of GREML-MS are consistent with GREML-KIN. The total contribution of all SNPs resulted in a heritability estimate of $50 \%(\mathrm{SE}=10 \%)$ for intelligence and $37 \%(\mathrm{SE}=10 \%)$ for education (Table 3$)$. This trend for the total heritability estimate derived from GREML-MS being similar to, but lower than, the heritability estimates derived from summing the $\mathrm{G}$ and $\mathrm{K}$ from GREML-KIN, and those derived from traditional pedigreebased methods (Fig. 2) was evident across all cognitive variables. This attenuation is consistent with the findings of Evans et al. [64] who showed that with imputation to HRC, GREML-MS can underestimate heritability by as much as variance. Bright red being common genetic effects captured by the $\mathrm{GRM}_{\mathrm{g}}$ matrix and dark red being the additional genetic effects captured by exploiting the higher level of linkage disequilibrium between family members using the $\mathrm{GRM}_{\text {kin }}$ matrix. Estimates shown in shades of blue were derived using GREML-MS and indicate the variance explained using unrelated individuals with genotyped data imputed to the HRC reference panel. The estimates in dark green are taken from Marioni et al. [63] and show the total genetic effects using ASReml-R mixed model when relatedness is inferred using identity by descent

$20 \%$ if the genetic architecture of a trait includes many rare variants.

When examining the variance explained by MAF using GREML-MS (Figs. 3 and 4) for general intelligence and education it is clear that the variants tagged by SNPs with a MAF between 0.001-0.01 make a large contribution to phenotypic variation. These low-MAF variants explain $23 \%$ $(\mathrm{SE}=10 \%)$ of the variation in intelligence, compared to $28 \%$ from variants with a MAF greater than 0.01 . For education, low-MAF variants explain $12 \%(\mathrm{SE}=10 \%)$, with all other variants explaining a total of $25 \%$. Similar findings were also evident for each of the cognitive tests used in the general intelligence phenotype (Supplementary Table 3). This was also found for extraversion, where variants with a MAF of 0.001-0.01 explained $17 \%(\mathrm{SE}=9 \%)$ whilst all other SNPs explained only $4 \%$ of phenotypic variance. However, for neuroticism there was no evidence of any contribution made by the SNPs with a MAF of 
Table 3 Results of GREML-MS variance components analyses for cognitive abilities and personality using six minor allele frequency cutoffs

\begin{tabular}{|c|c|c|c|c|c|c|c|c|}
\hline \multirow[b]{2}{*}{ Phenotype } & \multirow[b]{2}{*}{$N$} & \multicolumn{7}{|c|}{ Minor allele frequency (MAF) } \\
\hline & & $\begin{array}{l}0.001-0.01 \\
h^{2} \% \text { (S.E.) }\end{array}$ & $\begin{array}{l}>0.01-0.1 \\
h^{2} \% \text { (S.E.) }\end{array}$ & $\begin{array}{l}>0.1-0.02 \\
h^{2} \% \text { (S.E.) }\end{array}$ & $\begin{array}{l}>0.2-0.3 \\
\mathrm{~h}^{2} \% \text { (S.E.) }\end{array}$ & $\begin{array}{l}>0.3-0.4 \\
h^{2} \% \text { (S.E.) }\end{array}$ & $\begin{array}{l}>0.4-0.5 \\
h^{2} \% \text { (S.E.) }\end{array}$ & $\begin{array}{l}\text { Total variance } \\
\text { explained } \\
h^{2} \% \text { (S.E.) }\end{array}$ \\
\hline $\begin{array}{l}\text { Number of } \\
\text { SNPs }\end{array}$ & & $3,898,626$ & $3,320,146$ & $1,413,929$ & $1,061,603$ & 930,841 & 872,346 & $11,497,491$ \\
\hline \multicolumn{9}{|l|}{ Cognitive } \\
\hline$g$ & 7019 & $22.6(9.5)$ & $5.6(5.3)$ & $1.1(3.5)$ & $5.9(3.4)$ & $7.5(3.3)$ & $7.7(2.9)$ & $50.4(9.9)$ \\
\hline Education & 6860 & $12.1(9.6)$ & $1.5(5.2)$ & $4.0(3.6)$ & $9.3(3.6)$ & $9.0(3.4)$ & $1.32 .8)$ & $37.2(9.9)$ \\
\hline \multicolumn{9}{|l|}{ Personality } \\
\hline Neuroticism & 7195 & $1.0 \times 10^{-4}(8.8)$ & $3.6(5.0)$ & $1.0 \times 10^{-4}(3.2)$ & $2.3(2.9)$ & $0.9(2.9)$ & $4.7(2.6)$ & $11.4(9.4)$ \\
\hline Extraversion & 7188 & $17.0(9.2)$ & $1.0 \times 10^{-4}(4.7)$ & $1.0 \times 10^{-4}(3.2)$ & $1.1(3.1)$ & $1.1(3.0)$ & $1.8(2.5)$ & $20.9(9.6)$ \\
\hline
\end{tabular}

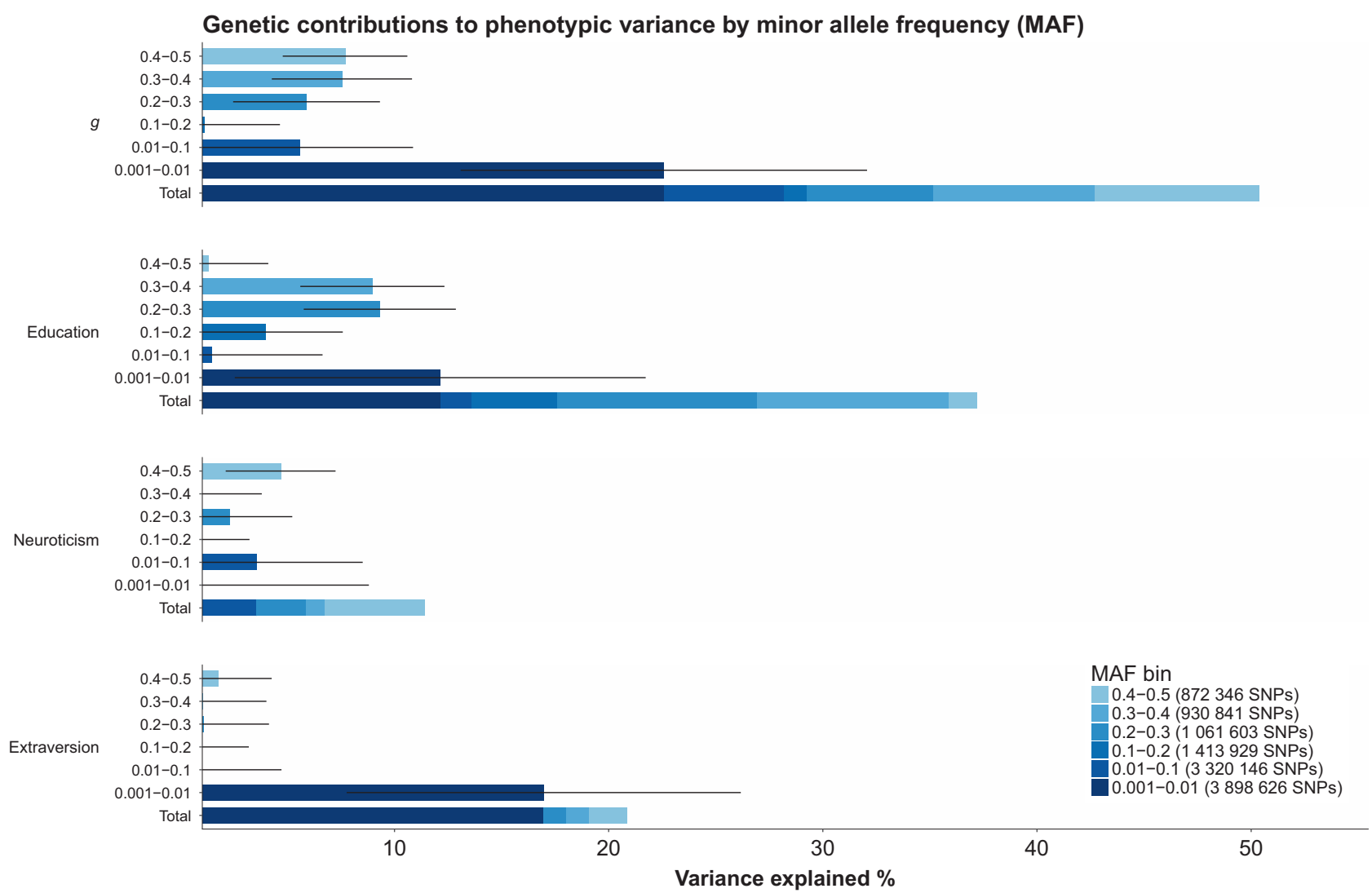

Fig. 3 Genetic contributions to each of the phenotypes by MAF derived using unrelated individuals and GREML-MS. Each MAF cutoff used is plotted separately, with the stacked bar plot showing the

$0.001-0.01$, and all variants only explained $11 \%(\mathrm{SE}=9 \%)$ of phenotypic variance.

We next examined if there was evidence of selective pressure acting on the cognitive and personality variables using GREML-MS. For a trait that is not under selective pressure while the majority of genetic variants will be rare, the total proportion of the variance explained by the each MAF cutoff. Error bars indicate standard error

majority of genetic variation associated with the trait is expected to be common [65]. A trait that is evolutionary neutral will, therefore, show a linear proportional relationship between MAF and cumulative genetic variance explained [62]. As can be seen in Fig. 4 general intelligence shows a deviation from the neutral evolutionary model. Education, an 


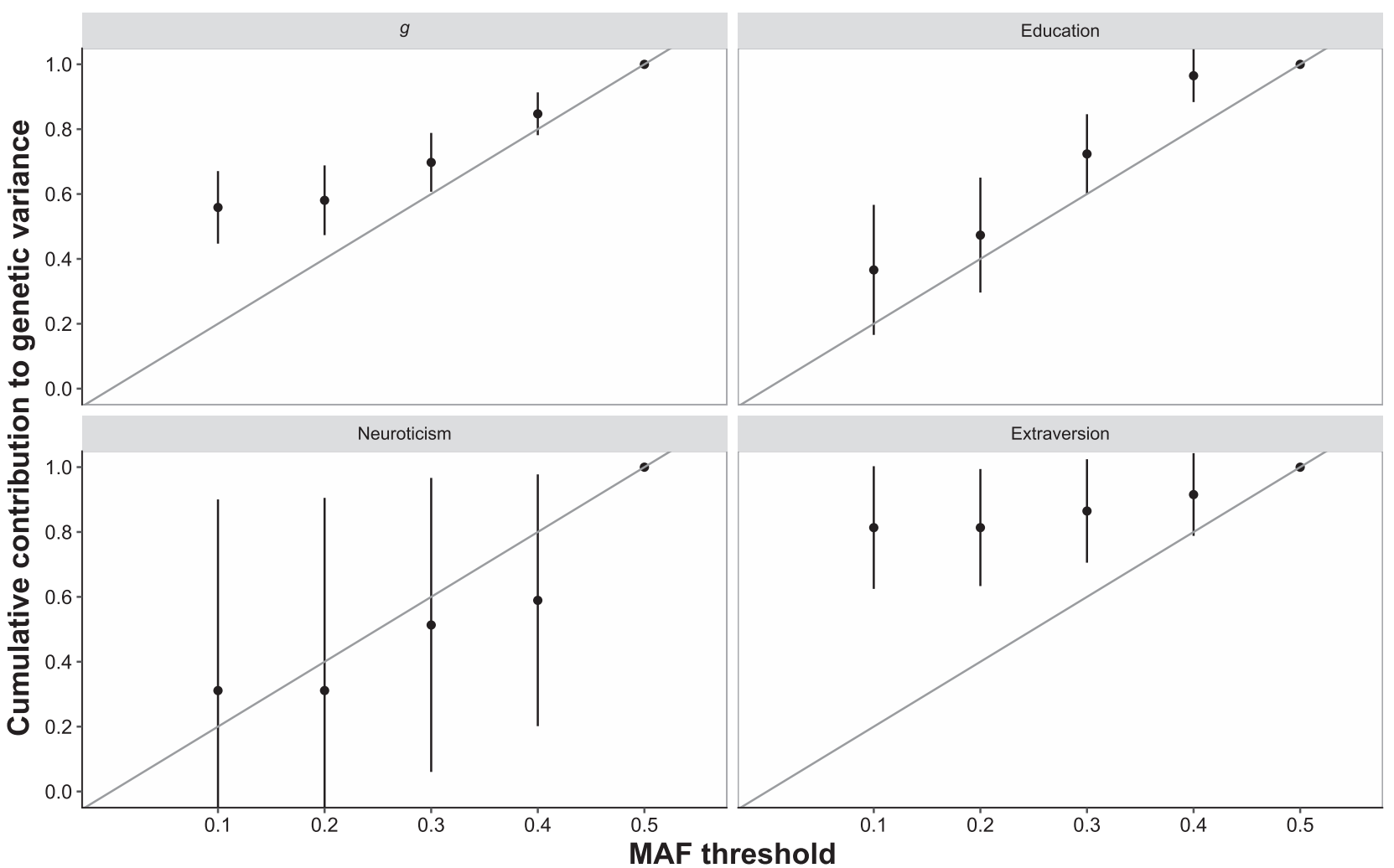

Fig. 4 MAF plotted against the cumulative genetic variance explained. The diagonal grey line indicates evolutionary neutrality where the proportion of genetic variance is proportional to the MAF. Error bars

often used proxy-phenotype for intelligence [42] showed no such deviation.

Extraversion also demonstrated evidence that low-MAF variants made a greater contribution than more common variants. Neuroticism, however followed the model predicted under the assumption of evolutionary neutrality.

\section{Discussion}

This study aimed to decompose and quantify additive genetic sources of variation to intelligence and personality in novel manners, using molecular genetic and pedigree data from the same large sample. In doing so, we sought to identify reasons for the gap between pedigree-based and SNP-based estimates of heritability in samples of unrelated individuals, a difference that might be due to genetic variants in poor LD with SNPs genotyped on current platforms. A number of novel findings speak to long-standing questions in behaviour genetics and evolutionary genetics of psychological differences [16, 32, 66].

Firstly, using GREML-KIN we could account for the entire heritability of general intelligence and education, as estimated in twin and family studies, by adding the $\mathrm{G}$ and $\mathrm{K}$ estimates we derived directly from genome-wide molecular represent standard errors for the cumulative variance components derived using the delta method, they are clipped if they leave the range of 0 to 1 [62]

genetic data [63, 67]. Secondly, using GREML-MS, we replicated this finding with imputed data on unrelated individuals. For general intelligence and education, a substantial and significant proportion of the phenotypic variance was found to be explained by pedigree-associated genetic effects $\left(h^{2}\right.$ kin $)$. The pedigree-associated genetic variants accounted for over half of the genetic effects in these phenotypes. Even though GREML-MS is expected to underestimate heritability for traits where the genetic architecture includes the contribution of CNVs, structural variants and very rare variants [64], we were nevertheless able to recover the majority of this heritability following imputation to the Haplotype Reference Consortium. For neuroticism, G plus K estimates were $\sim 30 \%$, even slightly exceeding the narrow-sense heritability estimates metaanalytically derived from family and adoption studies with heterogeneous measurements of personality [33]. However, the $\mathrm{K}$ component was dropped for extraversion in our model selection procedure. We believe that is due to the stringent statistical test, as described by Xia et al. if a component only explains $\sim 5 \%$ of the phenotypic variance in GS:SFHS, it might escape from model selection procedure (and $\mathrm{K}$ is $5 \%$ for extraversion). Furthermore, results were less consistent between GREML-KIN and GREML-MS for personality traits. These convergences and divergences between our 
two methods, and published results, are potentially diagnostic for the genetic architecture of the traits under study.

The GREML-SC method of estimating heritability from unrelated individuals using common genome-wide SNPs, often produces lower heritability estimates than those derived using family-based studies because it relies on LD between genotyped SNPs and causal variants at the population level. Should LD between genotyped SNPs and causal variants be low, then the genetic similarity between a pair of individuals at the causal variant will be different to the genetic similarity at genotyped SNPs, resulting in an underestimation of heritability. In within-family and twin studies, relatedness is based on identity by decent (IBD), where segments of DNA have been inherited from a recent common ancestor. Should a region be IBD between a pair of individuals, then all variants within that segment, except de novo mutations, are shared. Population-based SNP methods are sensitive to allele frequency, whereas IBD methods are blind to such effects. Therefore, the discrepancy between heritability estimates is consistent with the idea that causal variants in low LD with genotyped SNPs account for difference between IBD methods and population-based estimates derived using molecular genetic data.

In the current study, we investigate if variants in poor $\mathrm{LD}$ with genotyped SNPs account for additional heritability by using DNA from close family members. Higher genetic relatedness within families leads to an increase in the LD between genotyped SNPs and potentially causal variants, resulting in heritability estimates in our study that are comparable to pedigree-based methods. This provides evidence that for intelligence the gap between the heritability estimates derived using IBD methods and those derived using SNP-based population methods is most likely due to causal variants in low LD with genotyped SNPs. In addition, we were able to model this missing variance and separate it from the additive common genetic effects that are estimated in a GREML-SC analysis based on unrelated individuals. The additional source of additive genetic variance from closely related family members, captured here in our kinship matrix $\left(\mathrm{GRM}_{\mathrm{kin}}\right)$, would be unmeasured in a GWAS on unrelated individuals using genotyped data.

The use of related individuals can result in the confounding of pedigree genetic effects with shared family environmental effects. We were able to adjust for phenotype similarity driven by couple similarity, family similarity and sibling similarity, but some residual, uncorrected confounding might remain. Potential sources include geographical confounding, e.g., cousins attending the same school, and other environmental similarities that we could not adjust for. Such confounding was not modelled by Xia et al. and if present may represent a source of environmental variance still present in the genetic estimates of GREML-
KIN. However, previous work by Conley et al. [68] has shown that although environmental similarity can be correlated with relatedness, the effect this has on heritability is minor.

The three similarity matrices, $\mathrm{SRM}_{\mathrm{Sib}}, \mathrm{SRM}_{\mathrm{Family}}$ and $\mathrm{SRM}_{\text {Couple }}$ captured phenotypic similarity shared between siblings, nuclear family members and couples, respectively. The similarity shared between siblings is a product of additive genetic effects, dominance genetic effects, in addition to any environmental influences. However, by including the $\mathrm{GRM}_{\mathrm{g}}$ and the $\mathrm{GRM}_{\mathrm{kin}}$, what variance remained in $\mathrm{SRM}_{\mathrm{Sib}}$ is mainly due to environmental influences and some of the total dominance genetic effect as the additive genetic effects are captured by $\mathrm{GRM}_{\mathrm{g}}$ and $\mathrm{GRM}_{\mathrm{kin}}$. Similarly, SRM $_{\text {Family }}$ models similarity between nuclear family members, which is composed of additive genetic effects and environmental factors. In the presence of $\mathrm{GRM}_{\mathrm{g}}$ and $\mathrm{GRM}_{\text {kin }}$, what remained in $\mathrm{SRM}_{\text {Family }}$ will be variance attributable to environmental factors. The SRM Couple $_{1}$ represents the similarity between couples, which is mainly due to environmental influences, as well as the effects of assortative mating. However, the effect of couple environment and assortative mating are not confounded with the other matrices SRM's nor with either of the GRM's, because of this both the effects of assortative mating and the effects of any shared environmental influences acting to increase couple similarity will remain in the $\mathrm{SRM}_{\text {couple }}$.

It should be noted that, the average age of participants in GS:SFHS is 47.4-years-old, which means that the people still cohabiting together are most likely couples whereas parents-offspring and siblings no longer live in the same household. Additionally, dominance has been shown to have little impact on complex traits [69]. Therefore, in our selected model, the variance explained by $\mathrm{SRM}_{\mathrm{Sib}}$, $\mathrm{SRM}_{\text {Family }}$ and $\mathrm{SRM}_{\text {Couple }}$ will represent past environment shared by siblings and little dominance $\left(\mathrm{SRM}_{\mathrm{Sib}}\right)$, past environment shared by nuclear family members $\left(\mathrm{SRM}_{\text {Family }}\right)$ and assortative mating, in addition to potential current environmental factors shared by couples ( $\left.\mathrm{SRM}_{\text {Couple }}\right)$.

However, the replication of the GREML-KIN findings with GREML-MS in the subsample of unrelated individuals provides further evidence that the heritability estimates are not majorly affected by residual confounding. Indeed, for intelligence and education both of these methods provide highly similar estimates differing by $<4$ and 7 percentage points, respectively, well within one standard error of GREML-MS. These estimates in turn are highly similar to the estimate found using traditional pedigree-based analyses [63], indicating that the total narrow-sense heritability of intelligence can be captured using GREML-KIN. When using genotyped or imputed data, GREML-MS has been shown to underestimate the contribution made by rare variants to a polygenic traits by as much as $20 \%$ [64]. This 
is most likely due to the low imputation quality of rare SNPs, which can be ameliorated by using whole-genome sequencing data (WGS) to derive a heritability estimate. However, for traits where very rare variants have an effect (minor allele count $>5$ ), a downward bias is still apparent with WGS [64]. GREML-KIN can also capture non-SNPassociated variants like CNVs, which will also be missed by GREML-MS. This indicates that the accuracy of the heritability estimate provided by GREML-MS is dependent on the frequency of the causal variants that make up trait architecture, albeit much less so than using GREML-SC on genotyped data alone. Using GREML-KIN only a minor underestimation of heritability is seen in Evans et al. [64]. Supplementary Figs. 15 and 16 where regardless of MAF, heritability estimates are as accurate for genotyped data as they are for WGS. This suggests that, in the absence of environmental confounding, GREML-KIN approximates the true heritability better than GREML-MS. However, it should be noted that family-based analysis would be unsuitable for some phenotypes, such as those based on area or household measurements, as is the case with socioeconomic status or household income [70]. Converging estimates from the different methods increase our confidence in their interpretation as genetic effects, whereas the divergences between methods can help diagnose potential unmeasured sources contributing to broad-sense heritability or confounding.

The patterns found in our GREML-MS analyses were consistent with the findings of Evans et al. [64] for neuroticism and fluid intelligence. However, both GREML-KIN and GREML-MS estimates for neuroticism and extraversion fell short of estimates of broad-sense heritability in twin studies (47\% [33]; 45\% [71]). As previous research has suggested [33, 72], this is consistent with epistasis playing a major role in personality genetics, as a non-additive genetic component is not captured well outside of twin studies. Previous research [72] did not discuss gene-environment correlation and interaction as a plausible cause for heritability estimates being higher in twin than in adoption and family studies, presumably because the shared environment contribution to personality variation was usually estimated not to be different from zero. Still, the difference between twin estimates of heritability and those presented here may also be explained to some extent by gene-by-environment interactions and gene-environment correlations [32].

Another noteworthy divergence occurred between GREML-KIN and GREML-MS results for the personality traits. For extraversion, SNPs with a MAF of 0.001-0.01 explained $17.0 \% \quad(\mathrm{SE}=9.2)$ while the $\mathrm{K}$ component explained only $4.9 \%(\mathrm{SE}=5.1)$ and was dropped from the final selected model. However, the $\mathrm{G}$ plus $\mathrm{K}$ estimate for extraversion is $16.2 \%$, which is not significantly different from the total heritability estimate provided by GREMLMS (20.9\%). This is consistent with the interpretation that there is an effect of the $\mathrm{K}$ component for extraversion, which is too small to attain statistical significance in this sample. The results of neuroticism also do not match between GREML-KIN and GREML-MS. The total heritability estimate for GREML-MS was $11.4 \%$, similar to the $\mathrm{G}$ estimate, but in GREML-KIN the K explained a further $19 \%(\mathrm{SE}=2.5)$, while almost no effect was found for SNPs with a MAF of 0.001-0.01 using GREML-MS. As the GREML-KIN estimate is closer to twin and family study estimates of the narrow-sense heritability for neuroticism, this discrepancy might mean that the causal variants involved in neuroticism are even rarer, or perhaps due to non-SNPassociated genetic variants captured by GREML-KIN, but missed in GREML-MS. Potentially, the slightly lower measurement reliabilities for our personality measures may explain why results are less consistent than for intelligence.

The pattern we found using GREML-KIN is consistent with rare variants explaining much of the gap between heritability estimates from pedigree and GREML-SC analyses, although CNVs, and structural variation could also play a part, because they are poorly tagged by genotyped SNPs as well. This can be seen in Evans et al. [64], who used two genomic matrices, corresponding to the $\mathrm{GRM}_{\mathrm{g}}$ and the $\mathrm{GRM}_{\text {kin }}$ in the current study (for continuity, we will use our terms to describe their matrices). By varying the frequency of the causal variants in a simulated data set, Evans et al. showed that even when using only array markers, the total variance captured by these two matrices was equal to the true heritability in the data set, irrespective of the frequency of the causal variants. Consistent with the notion that the pedigree genetic effects captured by the $\mathrm{GRM}_{\mathrm{kin}}$ are due to the effect of rare variants, $\mathrm{GRM}_{\mathrm{kin}}$ captured an increasingly greater proportion of variance as the causal variant frequency fell. The reverse was true for the $\mathrm{GRM}_{\mathrm{g}}$, which captured less variance as causal variant frequency fell.

We found further, more direct support for an important role of rare variants using GREML-MS, which showed that for each of the cognitive variables examined here, a large contribution to phenotypic variance was made by SNPs with a MAF between 0.001 and 0.01 . For extraversion, almost all of the heritability was tagged by low-MAF SNPs. Altogether this indicates that the genetic signal to be found in imputed GWAS is much larger than GREML-SC estimates based on genotyped unrelated individuals would suggest.

In our GREML-MS results for general intelligence and extraversion the relationship between MAF and cumulative genetic variance explained was not proportionately linear, with increasing contributions being made to the genetic variance explained as MAF fell. This pattern contradicts the neutral evolutionary model [65] and suggests that rarer variants have a larger effect on intelligence and extraversion. This is consistent with previous findings that genetic variance in regions of the genome that have undergone 
purifying selection also make the greatest contributions to intelligence differences [24].

The GREML-KIN results favour the inclusion of a large $\mathrm{K}$ component for all traits except extraversion. This is consistent with a major contribution by rare and other poorly tagged variants. Previous work has already suggested a role for mutation-selection balance acting on harm avoidance and novelty seeking [73], traits that are related to neuroticism and extraversion, respectively [74].

A limitation of this the GREML-KIN approach is that Xspecific variance will go unnoticed. This is due to males being haploid and females being diploid at these regions and so the expected relationship on $\mathrm{X}$ chromosome is different between pairs of individual of the same sex and pairs of individuals of different sexes, even though they share the same degree of relationship, e.g., $\sim 0.5$ for mothers-and-daughters but 0 for fathers-and-sons. As all unmodelled variance remains in the residuals, the majority of the variance due to the $\mathrm{X}$ chromosome will, therefore, remain in the residuals.

Another limitation is that the variance analyses are blind to the direction of effects and the number of variants involved in each genetic component. If, as we would predict, future work finds that variants with the lowest minor allele frequencies tend to have larger negative effects on intelligence, it would imply a coupling between the selection coefficient of alleles and their effect on intelligence, as selective pressure would act to minimise the frequency of highly deleterious variants. If this coupling were strong [75], future work might infer that selection on intelligence was important in the past, even though current selective pressure appears to go in the opposite direction [76]. If the impact of intelligence on fitness were limited to instances of pleiotropy with, for example, health, as some initial research suggests [20, 21], the coupling between the selection coefficients of alleles and their effect sizes would be expected to be weaker. Selective pressure would act on the health-linked variants, whereas intelligence-linked variants would only be selected to the extent of their pleiotropic effects on health. This would de-couple the selection coefficient of an allele and its effect on intelligence. Therefore, such analyses could disentangle how much directly or indirectly intelligence has been under selection. Future work can use the SNPs known to affect intelligence and personality $[17,18]$ to empirically quantify the coupling between allele frequency (indicating selection strength) and effect size in order to test this explanation directly, as has been demonstrated for height and BMI [62]. Targeted resequencing of enriched genetic regions [24, 77, 78] might be necessary to find very rare genetic variants associated with intelligence and personality, as has proven fruitful for example in prostate cancer research [79].

The sibling similarity component, which was retained in all models of intelligence, tracks the meta-analytic estimate of shared environmental variance (11\%) from twin studies almost exactly. However, in our study the sibling component might also include the quarter of the dominance variation that siblings share, because siblings are the only relationship in this study where dominance plays a significant role [44]. In the classical twin design, dominance variation (making dizygotic twins more dissimilar than half the similarity of monozygotic twins) can be obscured by shared environment effects (making dizygotic twins more similar). There is some evidence from other approaches that dominance only plays a minor role in intelligence differences [80-83].

The family similarity component was only retained in the model for extraversion, although the point estimate was non-zero in the full neuroticism model as well. This is consistent with meta-analytic estimates of shared environment for adults [71]. However, it may also be due to some level of confounding between $\mathrm{K}$ and $\mathrm{F}$, where the association between extraversion and the $\mathrm{F}$ is due to contributions of the genetic factors accounted for by the $\mathrm{K}$.

The couple similarity component is somewhat complex to interpret. For intelligence and education, there is evidence of assortative mating [84], which will increase both the genetic and environmental similarity between couples. The couple similarity component may mostly reflect this spousal similarity, and possibly also the effects of more recent environmental influences. Beyond that, intelligence is not perfectly stable across the life course and studies of twins in earlier childhood frequently find a sizeable shared environment component. The importance of shared environment is usually said to decline from childhood to adulthood [85], as individuals pick their environmental individual niches (i.e., active gene-environment correlation), but this is based only on environment shared with siblings. However, it may also be that the current environment remains important and that the spouse is a better aggregated indicator of the current environment than the sibling with whom one usually no longer shares a home in adulthood. We find no couple similarity component for personality, which is consistent with much weaker assortative mating on personality, especially neuroticism and extraversion [86-88].

In the current study, we were able to exploit the high LD found between members of the same family to estimate the contribution of genetic effects that are normally missed in GREML-SC analyses of GWAS data. Using GREML-KIN, we simultaneously modelled the effect of the family, sibling and couple similarity to avoid potential environmental confounds inflating our estimates of the genetic effects. For intelligence and education, we find that genetic variants poorly tagged on current genotyping platforms explained a substantial proportion of the phenotypic variance, raising our heritability estimates to match those derived using pedigreebased quantitative methods. Such variants can include CNVs, structural variants, and rare variants. We find similar effects 
for neuroticism. For extraversion, pedigree-associated variants appear to play a smaller role in phenotypic variation. GREML-MS analyses, used with data imputed to the HRC reference panel, allowed us to examine lower frequency variants in a sample of unrelated individuals and provides strong convergent evidence, especially for intelligence and educational attainment. These results indicate that future GWAS using HRC imputation will be successful in finding the large majority of variants associated with intelligence. However for neuroticism whole-genome sequencing is likely to be more successful as our results from GREMLKIN suggest a large contribution from non-SNP/very rare/ poorly tagged genetic variants. Finally, our results suggest mutation-selection balance has maintained heritable variation in intelligence, and potentially to some degree also in neuroticism and extraversion, explaining why differences in these traits persist to this day despite selection. Future work should directly measure rare variants, as well as CNVs and structural variants, and test the direction of their effects.

Acknowledgements This work was undertaken in The University of Edinburgh Centre for Cognitive Ageing and Cognitive Epidemiology (CCACE), supported by the cross-council Lifelong Health and Wellbeing initiative (MR/K026992/1). Funding from the Biotechnology and Biological Sciences Research Council (BBSRC), the Medical Research Council (MRC) and the University of Edinburgh is gratefully acknowledged. CCACE funding supports IJD. WDH is supported by a grant from Age UK (Disconnected Mind Project). CSH, PN, CA and CX acknowledge MRC UK for funding (grants MC_PC_U127592696 and MC_PC_U127561128). CX is funded by the MRC and the University of Edinburgh. Generation Scotland received core support from the Chief Scientist Office of the Scottish Government Health Directorates [CZD/ 16/6] and the Scottish Funding Council [HR03006]. Genotyping of the GS:SFHS samples was carried out by the Genetics Core Laboratory at the Wellcome Trust Clinical Research Facility, Edinburgh, Scotland and was funded by the Medical Research Council UK and the Wellcome Trust (Wellcome Trust Strategic Award "STratifying Resilience and Depression Longitudinally" (STRADL) Reference 104036/Z/14/Z) to AMM, IJD, CSH and DP. RCA and LP acknowledge support from the Bielefeld Centre for Interdisciplinary Research group "Genetic and social causes of life chances".

\section{Compliance with ethical standards}

Conflict of interest The authors declare that they have no conflict of interest.

Open Access This article is licensed under a Creative Commons Attribution 4.0 International License, which permits use, sharing, adaptation, distribution and reproduction in any medium or format, as long as you give appropriate credit to the original author(s) and the source, provide a link to the Creative Commons license, and indicate if changes were made. The images or other third party material in this article are included in the article's Creative Commons license, unless indicated otherwise in a credit line to the material. If material is not included in the article's Creative Commons license and your intended use is not permitted by statutory regulation or exceeds the permitted use, you will need to obtain permission directly from the copyright holder. To view a copy of this license, visit http://creativecommons. org/licenses/by/4.0/.

\section{References}

1. Spearman C. "General Intelligence," objectively determined and measured. Am J Psychol. 1904;15:201-92.

2. Lahey BB. Public health significance of neuroticism. Am Psychol. 2009;64:241.

3. McCarthy S, Das S, Kretzschmar W, Delaneau O, Wood AR, Teumer A, et al. A reference panel of 64,976 haplotypes for genotype imputation. Nat Genet. 2016;48:1279-83.

4. Nagy R, Boutin TS, Marten J, Huffman JE, Kerr SM, Campbell A, et al. Exploration of haplotype research consortium imputation for genome-wide association studies in 20,032 Generation Scotland participants. Genome Med. 2017;9:23.

5. Posthuma D, De Geus EJC, Boomsma DI. Perceptual speed and IQ are associated through common genetic factors. Behav Genet. 2001;31:593-602.

6. Deary IJ. Intelligence. Annu Rev Psychol. 2012;63:453-82.

7. Davies G, Tenesa A, Payton A, Yang J, Harris SE, Liewald D, et al. Genome-wide association studies establish that human intelligence is highly heritable and polygenic. Mol Psychiatry. 2011;16:996-1005.

8. Kirkpatrick RM, McGue M, Iacono WG, Miller MB, Basu S. Results of a "GWAS Plus:" General cognitive ability is substantially heritable and massively polygenic. PLoS ONE. 2014;9:e112390.

9. Davies G, Marioni RE, Liewald DC, Hill WD, Hagenaars SP, Harris SE, et al. Genome-wide association study of cognitive functions and educational attainment in UK Biobank $(\mathrm{N}=112$ 151). Mol Psychiatry. 2016;21:758-67.

10. Batty GD, Deary IJ, Gottfredson LS, Premorbid (early life) IQ and later mortality risk: systematic review. Ann Epidemiol. 2007;17: 278-88.

11. Barban N, Jansen R, de Vlaming R, Vaez A, Mandemakers JJ, Tropf FC, et al. Genome-wide analysis identifies 12 loci influencing human reproductive behavior. Nat Genet. 2016;48:1462-72.

12. Day FR, Helgason H, Chasman DI, Rose LM, Loh P-R, Scott RA, et al. Physical and neurobehavioral determinants of reproductive onset and success. Nat Genet. 2016;48:617-23.

13. Deary IJ, Strand S, Smith P, Fernandes C. Intelligence and educational achievement. Intelligence. 2007;35:13-21.

14. Banks GC, Batchelor JH, McDaniel MA. Smarter people are (a bit) more symmetrical: A meta-analysis of the relationship between intelligence and fluctuating asymmetry. Intelligence. 2010;38:393-401.

15. Van Dongen S, Gangestad SW. Human fluctuating asymmetry in relation to health and quality: a meta-analysis. Evol Human Behav. 2011;32:380-98.

16. Penke L, Denissen JJ, Miller GF. The evolutionary genetics of personality. Eur J Personal. 2007;21:549-87.

17. Davies G, Armstrong N, Bis JC, Bressler J, Chouraki V, Giddaluru $\mathrm{S}$, et al. Genetic contributions to variation in general cognitive function: a meta-analysis of genome-wide association studies in the CHARGE consortium ( $N=53$ 949). Mol Psychiatry. 2015;20: 183-92.

18. Okbay A, Beauchamp JP, Fontana MA, Lee JJ, Pers TH, Rietveld $\mathrm{CA}$, et al. Genome-wide association study identifies 74 loci associated with educational attainment. Nature. 2016;533:539-42.

19. Simons YB, Turchin MC, Pritchard JK, Sella G. The deleterious mutation load is insensitive to recent population history. Nat Genet. 2014;46:220.

20. Bulik-Sullivan B, Finucane HK, Anttila V, Gusev A, Day FR, Loh P-R, et al. An atlas of genetic correlations across human diseases and traits. Nat Genet. 2015;47:12236-41.

21. Hill WD, Davies G, The CHARGE Cognitive Working Group, Liewald DC, McIntosh AM, Deary IJ. Age-dependent pleiotropy between general cognitive function and major psychiatric disorders. Biol Psychiatry. 2015;80:266-73. 
22. Beauchamp JP. Genetic evidence for natural selection in humans in the contemporary United States. Proc Natl Acad Sci. 2016;113: 7774-9.

23. Conley D, Domingue B. The bell curve revisited: Testing controversial hypotheses with molecular genetic data. Sociol Sci. 2016;3:520-39.

24. Hill WD, Davies G, Harris SE, Hagenaars SP, Liewald D, Penke $\mathrm{L}$, et al. Molecular genetic aetiology of general cognitive function is enriched in evolutionarily conserved regions. Transl Psychiatr. 2016;6:1-6.

25. Marioni RE, Penke L, Davies G, Huffman JE, Hayward C, Deary IJ. The total burden of rare, non-synonymous exome genetic variants is not associated with childhood or late-life cognitive ability. Proc R Soc B: Biol Sci. 2014;281:20140117.

26. MacLeod AK, Davies G, Payton A, Tenesa A, Harris SE, Liewald $\mathrm{D}$, et al. Genetic copy number variation and general cognitive ability. PLoS ONE. 2012;7:e37385.

27. McRae AF, Wright MJ, Hansell NK, Montgomery GW, Martin NG. No association between general cognitive ability and rare copy number variation. Behav Genet. 2013;43:202-7.

28. Kirkpatrick RM, McGue M, Iacono WG, Miller MB, Basu S, Pankratz N. Low-frequency copy-number variants and general cognitive ability: No evidence of association. Intelligence. 2014;42:98-106.

29. Luciano M, Svinti V, Campbell A, Marioni RE, Hayward C, Wright $\mathrm{AF}$, et al. Exome sequencing to detect rare variants associated with general cognitive ability: A pilot study. Twin Res Human Genet. 2015;18:117-25.

30. Yeo RA, Gangestad SW, Liu J, Calhoun VD, Hutchison KE. Rare copy number deletions predict individual variation in intelligence. PLoS ONE. 2011;6:e16339.

31. Ganna A, Genovese G, Howrigan DP, Byrnes A, Kurki MI, Zekavat SM, et al. Ultra-rare disruptive and damaging mutations influence educational attainment in the general population. Nat Neurosci. 2016;19:1563-5.

32. Penke L, Jokela M. The evolutionary genetics of personality revisited. Curr Opin Psychol. 2016;7:104-9.

33. Vukasović T, Bratko D. Heritability of personality: A metaanalysis of behavior genetic studies. Psychol Bull. 2015;141:769.

34. Smith DJ, Escott-Price V, Davies G, Bailey ME, Colodro-Conde L, Ward J, et al. Genome-wide analysis of over 106000 individuals identifies 9 neuroticism-associated loci. Mol Psychiatry. 2016;21:749-57.

35. van Den Berg SM, de Moor MH, Verweij KJ, Krueger RF, Luciano M, Vasquez AA, et al. Meta-analysis of genome-wide association studies for extraversion: Findings from the genetics of personality consortium. Behav Genet. 2016;46:170-82.

36. Lo M-T, Hinds DA, Tung JY, Franz C, Fan C-C, Wang Y, et al. Genome-wide analyses for personality traits identify six genomic loci and show correlations with psychiatric disorders. Nat Genet. 2016;49:152-6.

37. Terracciano A, Löckenhoff CE, Zonderman AB, Ferrucci L, Costa PT Jr. Personality predictors of longevity: activity, emotional stability, and conscientiousness. Psychosom Med. 2008;70:621.

38. Bienvenu OJ, Samuels JF, Costa PT, Reti IM, Eaton WW, Nestadt G, Anxiety and depressive disorders and the five-factor model of personality: A higher-and lower-order personality trait investigation in a community sample. Depress Anxiety. 2004;20:92-7.

39. Jokela M. Birth-cohort effects in the association between personality and fertility. Psychol Sci. 2012;23:835-41.

40. Roberts RO, Geda YE, Knopman DS, Cha RH, Pankratz VS, Boeve BF, et al. The incidence of MCI differs by subtype and is higher in men The Mayo Clinic Study of Aging. Neurology. 2012;78:342-51.
41. Krapohl E, Euesden J, Zabaneh D, Pingault J, Rimfeld K, Von Stumm S, et al. Phenome-wide analysis of genome-wide polygenic scores. Mol Psychiatry. 2015; https://doi.org/10.1038/mp. 2015.126.

42. Rietveld CA, Esko T, Davies G, Pers TH, Turley P, Benyamin B, et al. Common genetic variants associated with cognitive performance identified using the proxy-phenotype method. Proc Natl Acad Sci. 2014;111:13790-4.

43. Zaitlen N, Kraft P, Patterson N, Pasaniuc B, Bhatia G, Pollack S, et al. Using extended genealogy to estimate components of heritability for 23 quantitative and dichotomous traits. PLoS Genet. 2013;9:e1003520.

44. Xia C, Amador C, Huffman J, Trochet H, Campbell A, Porteous $\mathrm{D}$, et al. Pedigree-and SNP-associated genetics and recent environment are the major contributors to anthropometric and cardiometabolic trait variation. PLoS Genet. 2016;12:e1005804.

45. Smith BH, Campbell H, Blackwood D, Connell J, Connor M, Deary IJ, et al. Generation Scotland: the Scottish Family Health Study; a new resource for researching genes and heritability. BMC Med Genet. 2006;7:9.

46. Smith BH, Campbell A, Linksted P, Fitzpatrick B, Jackson C, Kerr SM, et al. Cohort profile: Generation Scotland: Scottish Family Health Study (GS: SFHS). The study, its participants and their potential for genetic research on health and illness. Int $\mathbf{J}$ Epidemiol. 2012;42:689-700.

47. Kerr SM, Campbell A, Murphy L, Hayward C, Jackson C, Wain $\mathrm{LV}$, et al. Pedigree and genotyping quality analyses of over 10,000 DNA samples from the Generation Scotland: Scottish Family Health Study. BMC Med Genet. 2013;14:38.

48. Gunderson KL. Whole-genome genotyping on bead arrays. In: Dufva M (ed). DNA Microarrays for Biomedical Research. New York: Humana Press; 2009, p. 197-213.

49. Amador C, Huffman J, Trochet H, Campbell A, Porteous D, Wilson JF, et al. Recent genomic heritage in Scotland. BMC Genom. 2015;16:1.

50. Raven JC, Court JH, Raven J. Manual for Raven's Progressive Matrices and Vocabulary Scales. London: H.K. Lewis; 1977.

51. Raven JC, Court JH. Raven's progressive matrices and vocabulary scales. UK: Oxford Psychologists Press Oxford; 1998.

52. Wechsler D. Wechsler Adult Intelligence Scale-3rd ed. London: The Psychological Corporation; 1998.

53. Wechsler D. Wechsler Memory Scale III UK. Psychological Corporation: London, 1998.

54. Lezak M, Howieson DB, Loring DW. Neuropsychological assessment. 4 ed. New York, NY: Oxford University Press, USA; 2004.

55. Eysenck SB, Eysenck HJ, Barrett $\mathrm{P}$, A revised version of the psychoticism scale. Personal Individ Differ. 1985;6:21-9.

56. Yang J, Lee SH, Goddard ME, Visscher PM. GCTA: a tool for genome-wide complex trait analysis. Am J Human Genet. 2011;88:7.

57. Yang J, Benyamin B, McEvoy BP, Gordon S, Henders AK, Nyholt DR, et al. Common SNPs explain a large proportion of the heritability for human height. Nat Genet. 2010;42:4.

58. Delaneau O, Zagury J-F, Marchini J. Improved wholechromosome phasing for disease and population genetic studies. Nat Methods. 2013;10:5-6.

59. Delaneau O, Marchini J, Zagury J-F. A linear complexity phasing method for thousands of genomes. Nat Methods. 2012;9: 179-81.

60. O'Connell J, Gurdasani D, Delaneau O, Pirastu N, Ulivi S, Cocca $\mathrm{M}$, et al. A general approach for haplotype phasing across the full spectrum of relatedness. PLoS Genet. 2014;10:e1004234.

61. Durbin R. Efficient haplotype matching and storage using the positional Burrows-Wheeler transform (PBWT). Bioinformatics. 2014;30:1266-72. 
62. Yang J, Bakshi A, Zhu Z, Hemani G, Vinkhuyzen AA, Lee SH, et al. Genetic variance estimation with imputed variants finds negligible missing heritability for human height and body mass index. Nat Genet. 2015;47:1114-20.

63. Marioni RE, Davies G, Hayward C, Liewald D, Kerr SM, Campbell A, et al. Molecular genetic contributions to socioeconomic status and intelligence. Intelligence. 2014;44:26-32.

64. Evans L, Tahmasbi R, Vrieze S, Abecasis G, Das S, Bjelland D, et al. Comparison of methods that use whole genome data to estimate the heritability and genetic architecture of complex traits. bioRxiv 2017: 115527.

65. Visscher P, Goddard M, Derks E, Wray N. Evidence-based psychiatric genetics, AKA the false dichotomy between common and rare variant hypotheses. Mol Psychiatry. 2012;17: 474-85.

66. Arslan RC, Penke L. Zeroing in on the genetics of intelligence. J Intell. 2015;3:41-5.

67. Chipuer HM, Rovine MJ, Plomin R. LISREL modeling: Genetic and environmental influences on IQ revisited. Intelligence. 1990;14:11-29.

68. Conley D, Siegal ML, Domingue BW, Harris KM, McQueen MB, Boardman JD. Testing the key assumption of heritability estimates based on genome-wide genetic relatedness. J Hum Genet. 2014;59:342-45.

69. Zhu Z, Bakshi A, Vinkhuyzen AA, Hemani G, Lee SH, Nolte IM, et al. Dominance genetic variation contributes little to the missing heritability for human complex traits. Am J Human Genet. 2015;96:377-85.

70. Hill WD, Hagenaars SP, Marioni RE, Harris SE, Liewald DC, Davies G, et al. Molecular genetic contributions to social deprivation and household income in UK Biobank. Curr Biol. 2016;26:3083-89.

71. Polderman TJ, Benyamin B, de Leeuw CA, Sullivan PF, van Bochoven A, Visscher PM, et al. Meta-analysis of the heritability of human traits based on fifty years of twin studies. Nat Genet. 2015;47:702-09.

72. Plomin R, Corley R, Caspi A, Fulker DW, DeFries J. Adoption results for self-reported personality: Evidence for nonadditive genetic effects? J Personal Social Psychol. 1998;75:211.

73. Verweij KJ, Yang J, Lahti J, Veijola J, Hintsanen M, PulkkiRåback L, et al. Maintenance of genetic variation in human personality: Testing evolutionary models by estimating heritability due to common causal variants and investigating the effect of distant inbreeding. Evolution. 2012;66:3238-51.

74. Vinkhuyzen AA, Pedersen NL, Yang J, Lee SH, Magnusson PK, Iacono WG, et al. Common SNPs explain some of the variation in the personality dimensions of neuroticism and extraversion. Transl Psychiatry. 2012;2:e102.
75. Eyre-Walker A. Genetic architecture of a complex trait and its implications for fitness and genome-wide association studies. Proc Natl Acad Sci. 2010;107:1752-56.

76. Kong A, Frigge ML, Thorleifsson G, Stefansson H, Young AI, Zink F, et al. Selection against variants in the genome associated with educational attainment. Proc Natl Acad Sci. 2017;114: E727-32.

77. Hill WD, Davies G, van de Lagemaat LN, Christoforou A, Marioni RE, Fernandes CPD, et al. Human cognitive ability is influenced by genetic variation in components of postsynaptic signalling complexes assembled by NMDA receptors and MAGUK proteins. Transl Psychiatry. 2014;4:e341.

78. Johnson MR, Shkura K, Langley SR, Delahaye-Duriez A, Srivastava $\mathrm{P}$, Hill WD, et al. Systems genetics identifies a convergent gene network for cognition and neurodevelopmental disease. Nat Neurosci. 2015;19:223-232.

79. Mancuso N, Rohland N, Rand KA, Tandon A, Allen A, Quinque $\mathrm{D}$, et al. The contribution of rare variation to prostate cancer heritability. Nat Genet. 2015;48:30-35.

80. Bashi J. Effects of inbreeding on cognitive performance. Nature. 1977;266:440-2.

81. Gamsiz ED, Viscidi EW, Frederick AM, Nagpal S, Sanders SJ, Murtha MT, et al. Intellectual disability is associated with increased runs of homozygosity in simplex autism. Am J Human Genet. 2013;93:103-9.

82. Joshi PK, Esko T, Mattsson H, Eklund N, Gandin I, Nutile T, et al. Directional dominance on stature and cognition in diverse human populations. Nature. 2015;523:459-62.

83. Power AR, Nagoshi C, DeFries JC, Wellcome Trust Case Control Consortium, Plomin R. Genome-wide estimates of inbreeding in unrelated individuals and their association with cognitive ability. Eur J Hum Genet. 2014;22:386-90.

84. Jensen AR. The g factor: The science of mental ability. Westport, CT: Praeger; 1998.

85. Plomin R, Deary IJ. Genetics and intelligence differences: five special findings. Mol Psychiatry. 2014;20:98-108.

86. Dyrenforth PS, Kashy DA, Donnellan MB, Lucas RE. Predicting relationship and life satisfaction from personality in nationally representative samples from three countries: the relative importance of actor, partner, and similarity effects. J Personal Social Psychol. 2010;99:690.

87. Rammstedt B, Spinath FM, Richter D, Schupp J. Partnership longevity and personality congruence in couples. Personal Individ Differ. 2013;54:832-35.

88. Sherlock JM, Verweij KJ, Murphy SC, Heath AC, Martin NG, Zietsch BP. The role of genes and environment in degree of partner self-similarity. Behav Genet. 2016;47:25-35. 